\title{
A CLINICAL STUDY ON ACUTE OPHTHALMIC MANIFESTATION AND MANAGEMENT OF STEVENS- JOHNSON SYNDROME CONDUCTED AT A TERTIARY CENTRE OF THIRUVANANTHAPURAM, KERALA
}

\author{
S. Rema Devi', Ajith Chakravarthy²
}

${ }^{1}$ Assistant Professor, Regional Institute of Ophthalmology, Government Medical College Hospital, Thiruvananthapuram, Kerala, India. ${ }^{2}$ Lecturer, Department of Ophthalmology, Government Medical College Hospital, Thiruvananthapuram, Kerala, India.

ABSTRACT
BACKGROUND
SJS (Stevens-Johnson Syndrome) is an acute life-threatening immune complex mediated hypersensitivity reaction to drugs that
results in extensive necrosis and detachment of the epidermis, which shows widespread lesions affecting mouth, eyes, pharynx,
larynx, skin, genitals etc. Acute ocular manifestation caused by SJS causes severe ocular morbidities if not treated at an early stage.

\section{MATERIALS AND METHODS}

A descriptive follow-up study was undertaken at Regional Institute of Ophthalmology (RIO), Thiruvananthapuram, Southern Kerala. Patients presented at Dermatology and General Medicine Department of Medical College Hospital, Thiruvananthapuram, identified with SJS were examined and called for detailed ocular evaluation at RIO. A total of 52 cases were screened during August 2009 to 2011 and all patients were told about the severity of disease and their detailed drug history, personal history and ocular symptoms were collected. Protocol based treatments were offered to all subjects and effect of treatment was studied during follow-up periods. Data of the patients was recorded on a prefabricated proforma. Data was statistically analysed to find out association and comparison between different parameters using non-parametric Chi-square $\left(\chi^{2}\right)$ test.

\section{RESULTS}

$23.1 \%$ of SJS subjects showed toxicity to ciprofloxacin; $96.2 \%$ showed acute ophthalmic manifestation. Catarrhal type of conjunctivitis was the commonest, which responds well to terramycin eye ointment and frequent irrigation of eyes. Corneal erosions and corneal ulceration in the acute period were reduced in the follow-up period due to active ophthalmic intervention in the early period.

\section{CONCLUSION}

1. SJS is a life-threatening illness with high incidence of ocular involvement.

2. Conjunctivitis is the commonest acute ophthalmic manifestation.

3. Early interference and active treatment regimen can reduce the events and further extent of corneal involvement.

\section{KEYWORDS}

Stevens-Johnson Syndrome, Punctate Epithelial Erosion, Superficial Punctate Epithelial Erosion, Toxic Epidermal Necrolysis.

HOW TO CITE THIS ARTICLE: Devi SR, Chakravarthy A. A clinical study on acute ophthalmic manifestation and management of Stevens-Johnson Syndrome conducted at a tertiary centre of Thiruvananthapuram, Kerala. J. Evolution Med. Dent. Sci. 2018;7(19): 2420-2425, DOI: $10.14260 /$ jemds/2018/544

\section{BACKGROUND}

SJS (Stevens-Johnson Syndrome) is an acute life-threatening immune complex mediated hypersensitivity reaction to drugs that results in extensive necrosis and detachment of the epidermis which shows widespread lesions affecting mouth, eyes, pharynx, larynx, skin, genitals etc. SJS confirmed cases were studied at RIO, Thiruvananthapuram, prospectively and the data obtained were statistically analysed. Ocular manifestation in these studies were conjunctivitis, conjunctival bullae, symblepharon following membranous conjunctivitis and corneal erosions and corneal ulcerations, PEE (Punctate Epithelial Erosions), Superficial Punctate Epithelial Erosions (SPEE) and uncommonly cases of corneal opacity.

'Financial or Other Competing Interest': None.

Submission 19-03-2018, Peer Review 24-04-2018,

Acceptance 30-04-2018, Published 07-05-2018.

Corresponding Author:

Dr. S. Rema Devi,

PRA-16, T. C. 15/2997,

Pettah Post, Thiruvananthapuram-24,

Kerala.

E-mail: drremasreekanth@gmail.com

DOI: $10.14260 /$ jemds $/ 2018 / 544$

\section{(c) (i) $\$$}

Among the acute manifestations, Catarrhal type of conjunctivitis is the commonest followed by mucopurulent type and membranous type. In acute stage symblepharon, the involvement in lower lid was more and they broke on lysis with glass rod and a spatula. Patient with milder skin involvement may still have severe ophthalmologic inflammation and most need prompt and persistent ophthalmologic evaluation during the acute phase. If the patient is critically ill and require specialised nursing and medical care, careful monitoring is a must. Any potentially offending drugs should be discontinued immediately. Management is undertaken as soon as possible in acute cases and be admitted in intensive care units or in burn units. Proper fluid balance management is crucial because of the loss of stratum corneum. Ocular surface hygiene should be monitored with frequent conjunctival irrigation and instillation of prophylactic antibiotics to control secondary infection. Topical steroids were used to relieve scarring to an extent. Milder cases of non-membranous conjunctivitis and non-lid margin involvement or corneal involvement may be managed with daily inspection. In corneal involvement, cycloplegics can also be used to relieve pain, photophobia and ciliary spasm. Topical antibiotics and anti-inflammatory drugs and agents such as cyclosporine or corticosteroid drops may 
be used. To relieve Symblepharon rings or bandage, contact lens can be used to separate between the palpebral conjunctivae from bulbar conjunctivae. Use of lubricating eye drops itself in the acute phase is advocated by some doctors. Early interference and active treatment regimen in the acute phase itself can reduce or prevent in going for late phase ocular complications in this study.

\section{Aim}

1. To find out the acute ophthalmic manifestation of patients attending a tertiary eye care hospital in Southern Kerala and their management.

2. To find out the correlation between drug aetiology and ophthalmic manifestations.

\section{Settings}

Regional Institute of Ophthalmology (RIO), Government Medical College Hospital, Thiruvananthapuram, Kerala.

\section{MATERIALS AND METHODS}

The study was designed as a descriptive follow-up study and patients presented at Dermatology and General Medicines Department of Government Medical College Hospital, Thiruvananthapuram, identified with SJS were examined and called for detailed ocular evaluation at RIO. A total of 52 cases were screened during August 2009 to August 2011 after getting consent from Institutional Human Ethical Committee.

All patients were told about the severity of the disease and their detailed drug history, personal history and ocular symptoms were collected and subjected to detailed ocular examination with slit lamp evaluation of lids, conjunctivae and cornea and anterior chamber. Protocol based treatment were offered to all subjects and effect of treatment was evaluated during the $1^{\text {st }}$ visit and later follow-up periods. Vision acuity was noted initially and follow-up visits.

All patients were informed about the chronicity of the disease. Patient was called for follow-up visit for 2 weeks, 4 weeks, 2 months and 6 months. Data of the patients were recorded on a prefabricated Proforma. Data was statistically analysed to find out association and comparisons between different parameters using non-parametric Chi-square $\left(\chi^{2}\right)$ test. For all statistical evaluations, a two-tailed probability of value $\mathrm{p}<0.05$ was considered significant.

\section{RESULTS}

Ophthalmic manifestations of 52 SJS patients along southern Kerala, India were investigated, and the results are as given below.

\section{Age Distribution}

Age (years) group analysis of the population showed majority of the subjects were between 30 - 39 years (Table 1 ) and the mean age was $39.56 \pm 3.56$. SJS was seen commonly in the $3^{\text {rd }}$ and $6^{\text {th }}$ decade in this study.

\begin{tabular}{|c|c|c|}
\hline Age & Frequency & Percent \\
\hline$<20$ yrs. & 7 & 13.5 \\
\hline $20-29$ & 8 & 15.4 \\
\hline $30-39$ & 14 & 26.9 \\
\hline $40-49$ & 8 & 15.4 \\
\hline $50-59$ & 6 & 11.5 \\
\hline$>=60$ yrs. & 9 & 17.3 \\
\hline Total & $\mathbf{5 2}$ & $\mathbf{1 0 0}$ \\
\hline \multicolumn{2}{|c|}{ Table 1. Age Distribution } \\
\hline
\end{tabular}

\section{Causative Drugs}

In the present study, it showed Ciprofloxacin was effective in $12(23.1 \%)$ patients followed by paracetamol 9 (17.3\%) and Sulphamethoxazole 8 (15.4\%), which was followed by antiepileptics (Phenytoin) and Carbamazepine were effective in $6(11.5 \%)$ and $4(7.7 \%)$ patients respectively. NSAIDS (Brufen) as $3(5.8 \%)$ and other groups of drugs as 5 (9.6\%) (Table 2 and Fig. 2).

\begin{tabular}{|c|c|c|}
\hline Drug & Frequency & Percent \\
\hline No drug intake & 2 & 3.8 \\
\hline Ciprofloxacin & 12 & 23.1 \\
\hline Paracetamol & 9 & 17.3 \\
\hline Ibuprofen & 3 & 5.8 \\
\hline Sulphamethoxazole & 8 & 15.4 \\
\hline Allopurinol & 3 & 5.8 \\
\hline Carbamazepine & 4 & 7.7 \\
\hline Phenytoin & 6 & 11.5 \\
\hline Others & 5 & 9.6 \\
\hline Total & 52 & 100 \\
\hline \multicolumn{3}{|c|}{ Table 2. Table showing Causative Drugs } \\
\hline
\end{tabular}

\section{Ophthalmic Manifestations}

Ocular involvement was very high in this study. SJS has several ophthalmic manifestations, of which the conjunctival involvements are the major causative reasons for different disease complex or syndromes.
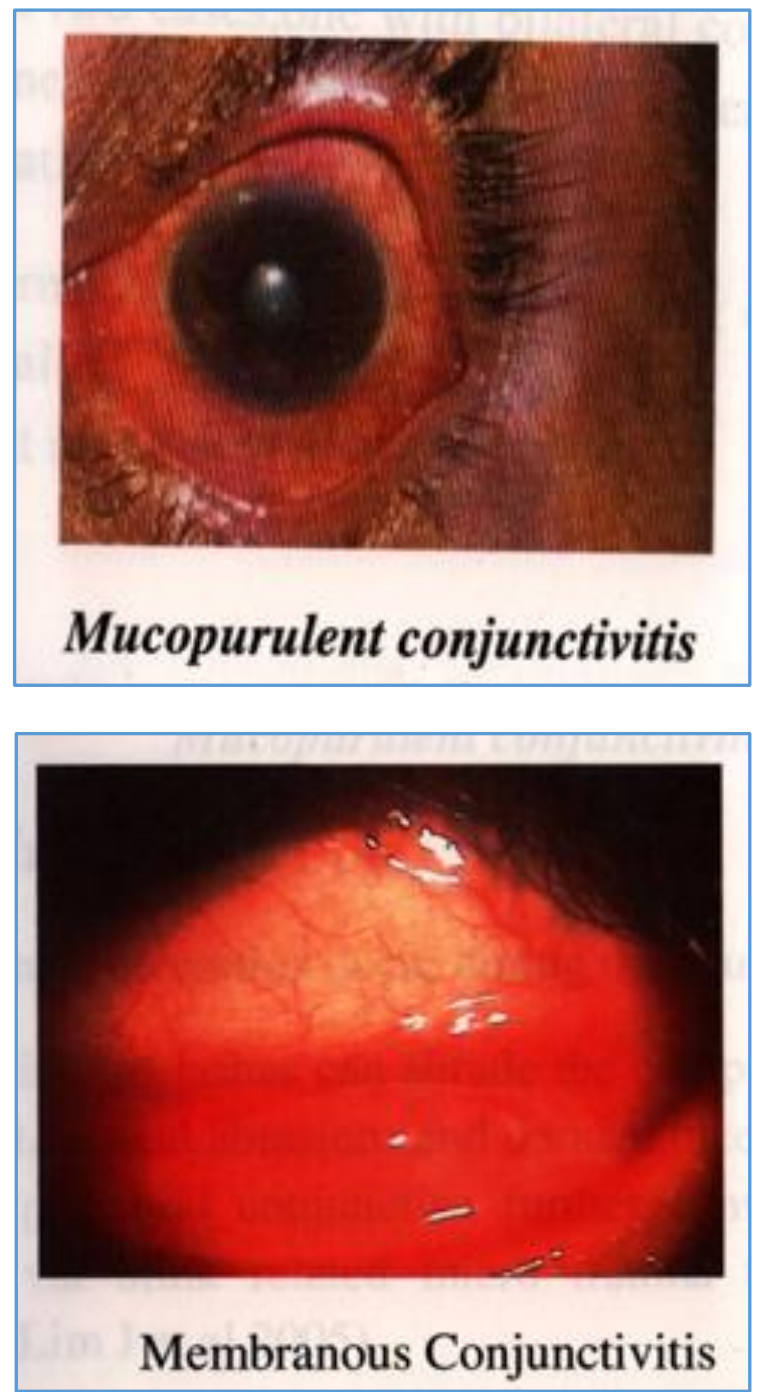


\section{Conjunctival Involvement in Acute Stage}

In the acute stage, conjunctivitis was the commonest ophthalmic manifestation and was seen in 50 patients (96.2\%) (Table 3 and Fig. 3).

\begin{tabular}{|c|c|c|}
\hline Acute Conjunctival Involvement & Frequency & Percent \\
\hline Catarrhal & 26 & 50.0 \\
\hline MPC & 10 & 19.2 \\
\hline Membranous conjunctivitis & 9 & 17.3 \\
\hline Others & 5 & 9.6 \\
\hline Total & 52 & 100 \\
\hline
\end{tabular}

Types of Conjunctivitis in the Acute Stage

In this study, commonest type of conjunctivitis is catarrhal conjunctivitis (50\%) (Table 4a).

$\begin{array}{lr}\text { Types of Conjunctivitis } & \text { Percent } \\ \text { MP } & 19.20 \% \\ \text { MC } & 17.30 \% \\ \text { Others } & 9.60 \% \\ \text { Absent } & 3.80 \% \\ \text { Catarrhal } & 50.00 \%\end{array}$

Laterality of Conjunctivitis in the Acute Stage

Laterality of conjunctivitis is bilateral, i.e. frequencies in Right and Left eye is $100 \%$ (Table $4 \mathrm{~b}$ ).

\begin{tabular}{|c|c|c|c|c|}
\hline \multirow{2}{*}{$\begin{array}{c}\text { Conjunctival } \\
\text { Involvement }\end{array}$} & \multicolumn{2}{|c|}{ Right Eye } & \multicolumn{2}{c|}{ Left Eye } \\
\cline { 2 - 5 } & Frequency & Percent & Frequency & Percent \\
\hline Absent & 2 & 3.8 & 0 & 0.0 \\
\hline Catarrhal & 26 & 50.0 & 28 & 53.8 \\
\hline MP & 10 & 19.2 & 10 & 19.2 \\
\hline MC & 9 & 17.3 & 9 & 17.3 \\
\hline Others & 5 & 9.6 & 5 & 9.6 \\
\hline Total & $\mathbf{5 2}$ & $\mathbf{1 0 0}$ & $\mathbf{5 2}$ & $\mathbf{1 0 0}$ \\
\hline \multicolumn{5}{|c|}{ Chi-square: 0.658; P > 0.05 } \\
\hline Table 4. Table showing Laterality of Conjunctivitis in the \\
Acute Stage \\
\hline
\end{tabular}

\section{Symblepharon in Acute Stage}

The present study showed symblepharon in 10 eyes and they broke on lysis with glass rod and a spatula and relieved by cutting the band. About the symblepharon involvement upper eye lid involvement in right eye as 3.8\% and left eye as 5.8\% and in lower eye lid involvement in right eye as $13.5 \%$ and left eye as $13.5 \%$ as similar to other hospital-based studies $(14 \%)$.

\begin{tabular}{|c|c|c|}
\hline Acute Symblepharon & RE & LE \\
\hline Absent & $82.70 \%$ & $80.80 \%$ \\
\hline Upper lid & $3.80 \%$ & $5.80 \%$ \\
\hline Lower lid & $13.5 \%$ & $13.5 \%$ \\
\hline \multicolumn{2}{|c|}{ Chi-square: 0.879; P > 0.05 } \\
\hline \multicolumn{2}{|c|}{ Table 5. Acute Symblepharon } \\
\hline
\end{tabular}

\section{Membranous Conjunctivitis}

9 patients showed membranous conjunctivitis (17.3\%), 5 patients showed other type of conjunctivitis (9.6\%).

\section{Corneal involvement in Acute Stage}

The present study showed corneal involvement in acute stage, also Corneal involvement erosions were shown by 9 (17.3\%) and Superficial Punctuate Epithelial Erosions (SPE) were shown by $7(13.6 \%)$ of the total study population. Punctuate Epithelial Erosions (PEE) were shown by 12 $(23.3 \%)$ and SPEE were shown by 7 (13.6\%).

Corneal opacity were noted in $3(5.7 \%)$ patients, uncommonly corneal opacity occur during acute phase. Among these, three cases of initial or very early ophthalmic interference could not succeed. The incidence of acute corneal corrosion presented in 9 patients were reduced to 3 in the follow-up period and 12 of patients presented with PEE were reduced to 5 in the follow-up period followed by SPEE. This shows active and early ophthalmologic intervention and meticulous treatment regime can reduce the corneal involvement to certain extent. Chi-square test conducted in this group $(p<0.001)$ showed significant reduction in the incidence of corneal involvement in the follow-up period.

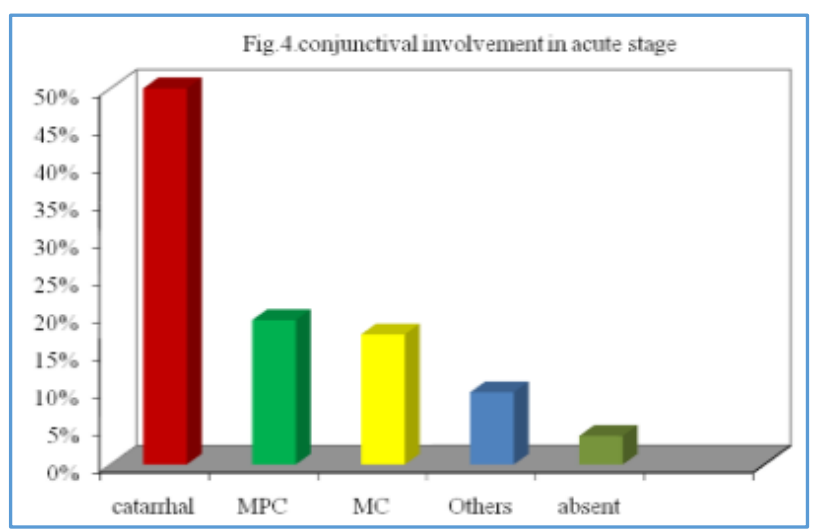

Figure 1. Acute Conjunctival Involvement
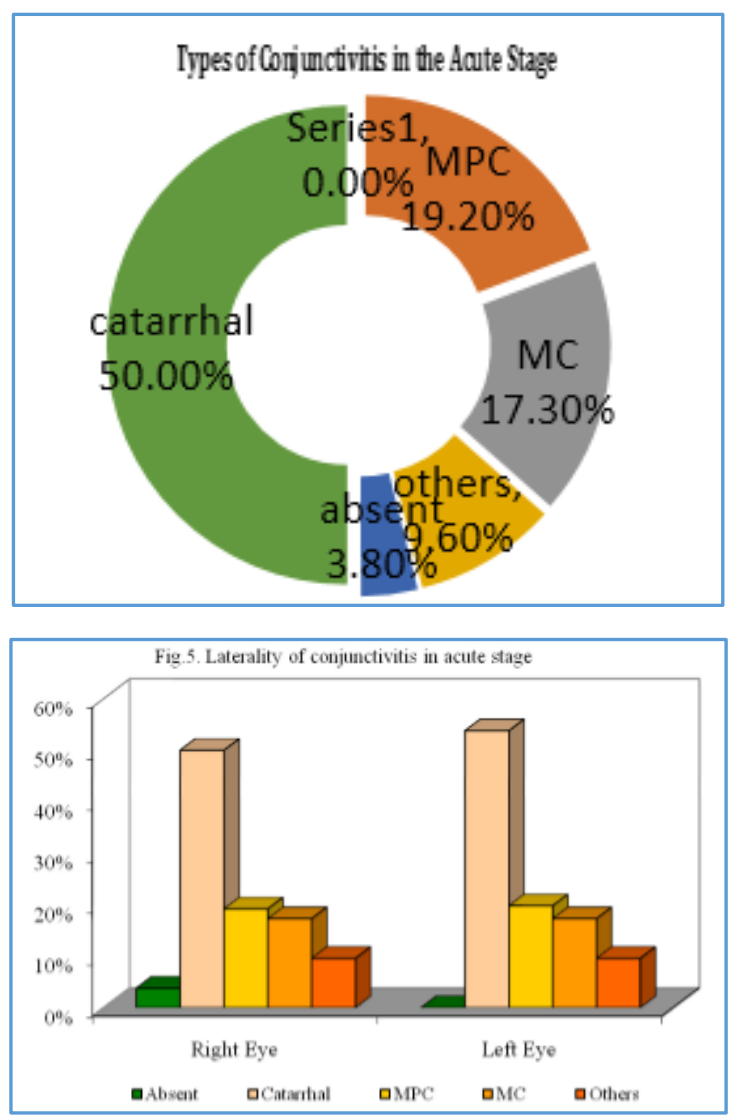


\begin{tabular}{|c|c|c|}
\hline Acute Corneal Involvement & Frequency & Percent \\
\hline PEE & 12 & $23.1 \%$ \\
\hline Erosion & 9 & $17.3 \%$ \\
\hline SPEE & 7 & $13.6 \%$ \\
\hline Corneal opacity & 3 & $5.7 \%$ \\
\hline Absent & 21 & $40.4 \%$ \\
\hline Total & $\mathbf{5 2}$ & $\mathbf{1 0 0} \%$ \\
\hline \multicolumn{2}{|c|}{}
\end{tabular}

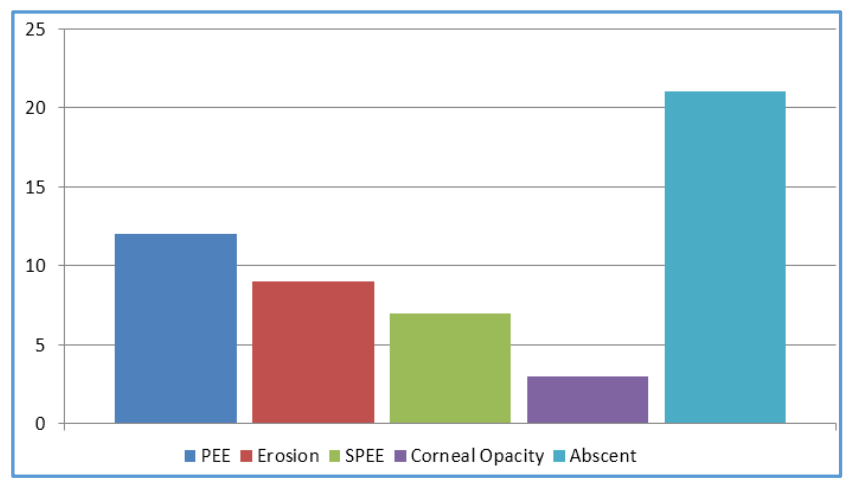

Figure 3

\section{DISCUSSION}

SJS causes mucosal erosions with widespread erythematous cutaneous macular or target lesions that merge together with subsequent epidermal detachment. Previous studies shown about drug responsibility is $55.99 \%$ of SJS. All age groups are affected, but incidence however appear to have peaked in the second (Thomas, 1950; Duggan and Gaines, 1951) and fifth decade. Approximately 6 cases/ million persons/ year were affected.1,2 (Schopf et al, 1991; Roujeau et al, 1995) reported an overall risk of 0.93 / millions persons/ year for Toxic epidermal necrolysis and 1.1 per million persons/ year for SJS. Again, Roujeau et al (1995) ${ }^{1}$ describes an incidence of 1.2 to 1.3 cases per million/ year for toxic epidermal necrolysis and 1.1 per million persons/ year for SJS.

Recent studies revealed genetic susceptibility to a certain extent as in some East-Asian population studies HLA Class I marker in Japanese patients with carbamazepine-induced cutaneous adverse reactions (Ikeda et al, 2010). ${ }^{3} \mathrm{~A}$ significant majority of patients with Acute SJS/ TEN will have ophthalmic pathology. According to Storm (1996), ${ }^{4}$ he found that eyes are very often affected $93 \%$ and called it as a mucocutaneous ocular fever. Studies by Power et al (2007) ${ }^{5}$ and Chang et al $(2011)^{6}$ reported ocular involvement in $69 \%$ and $81 \%$ respectively of patients with SJS and TEN. Yip $(2005)^{7}$ reported acute ocular involvement in $69 \%$ of SJS/ TEN patients. The type and severity of ocular involvement seems to have no relation to the extent of severity of systemic disease (Duggan and Gaines, 1951). ${ }^{8}$ The incidence of ocular involvement during the acute phase has been reported to be between $69 \%$ and $82 \%$ for SJS and $50 \%$ and $88 \%$ for TEN (Pushker et al, 2000; Power et al, 2007).5,9

Conjunctivitis is the most frequent involved structure in the eye in SJS (Thomas, 1950; Duggan and Gaines, 1951).8,10 Pohloja and Fleimo (1966) ${ }^{11}$ and Stewart et al (1994) ${ }^{12}$ reported conjunctivitis in $82 \%$ of cases of SJS. It is usually bilateral (Ashby and Lazar, 1951; Ting and Adam, 1985).13,14 The bilateral conjunctivitis may be catarrhal or pseudomembranous and occur in $15 \%$ to $75 \%$ patients with SJS (Bianchine et al, 1968). ${ }^{15}$
A more severe conjunctival lesions results in pseudomembranous or membranous conjunctivitis as reported by Foster et al (1988), ${ }^{16}$ which may often lead to catarrhal conjunctivitis. Commonest type of conjunctivitis is the catarrhal type. But few authors (Duggan and Gaines, 1951; Howard, 1963; Strom, 1966) $)^{8,17,4}$ states purulent conjunctivitis and pseudomembranous were the commonest manifestations. Misra and Chandi Das (1968) ${ }^{18}$ described a case of SJS with severe bilateral congestion of bulbar and tarsal congestion of conjunctivae with profuse mucopurulent discharge.

\section{Conjunctival Chemosis}

Reported by Power et al (2007) ${ }^{5}$ in acute phase.

\section{Lid-Globe Adhesions}

Power et al (2007) ${ }^{5}$ reported lid-globe adhesions in many cases of SJS.

\section{Conjunctival Bullae}

As an acute manifestation noticed by Ashby and Lazar (1951) ${ }^{13}$ and Champion et al (1998). ${ }^{19}$

\section{Corneal Involvement}

Corneal erosions and ulcerations occur in the acute stage of SJS and TEN reported by Howard (1963),17 Wilkins et al(1992) ${ }^{20}$ and Albert et al (1994). ${ }^{21}$ It can lead to perforation also (Shah et al, 1977).22 12 of 20 (60\%) cases of SJS reported by Sarkari et al (1972) ${ }^{23}$ had corneal involvement. Champion et al (1998) ${ }^{19}$ also described about corneal ulceration. Shaffer and Morris (1948) ${ }^{24}$ reported a case of corneal perforation occurred in one eye in acute phase and in two cases almost complete blindness resulted from corneal ulceration. Anterior uveitis occur in acute stage of SJS reported by Huff et al (1983) ${ }^{25}$ and Wilkins et al (1992).20

In the acute phase itself, active interference by some local measures plays an important role to prevent ocular morbidity to some extent.

Important role measure advocates include:- Tear film substitute, frequent irrigation of fornix, removal of pseudomembrane, lysis of symblepharon using glass rod and topical antibiotics to prevent secondary infection, topical steroids to prevent scar formation, topical cycloplegics to prevent pain, photophobia and ciliary spasm and corneal epithelial integrity by pad and bandage.

\section{Causative Drugs}

In the present study showed ciprofloxacin effective in 12 (23.1\%) patients followed by paracetamol 9 (17.3\%) and Sulphamethoxazole 8 (15.4\%), which was followed by antiepilepticus (Phenytoin) and Carbamazepine were effective in $6(11.5 \%)$ and $4(7.7 \%)$ patients respectively. NSAIDS (Brufen) as $3(5.8 \%)$ and other groups of drugs as $5(9.6 \%)$ (Table 2 and Fig. 2). Data from surveillance network cited by Roujeau et al (1995) ${ }^{1}$ on 245 people hospitalised for SJS reported risk factors was oral intake of $80 \%$ NSAIDS and similar observations were also reported by Storm (1966). ${ }^{4}$ But in this study, Brufen contributes only (5.8\%) as an aetiological factor and also mentioned about new users of antiepileptic adverse reactions as an aetiological factor. In the present study, antiepileptic contributes 19.2\%. A survey of selected patients with epilepsy over $80 \%$ has reported at least 
one adverse event and the majority had more than one adverse event (Pushker et al, 2000). ${ }^{9}$

\section{Ophthalmic Manifestations}

Ocular involvement was very high in this study. A retrospective study conducted at National Cheng King University Hospital in Taiwan between 1988 - 2004 attackers of SFS/ EM of 207 patients showed SJS in high ophthalmic involvement (81.3\%) (Yip, 2007). ${ }^{7}$

\section{Conjunctival Involvement in Acute Stage}

Stewart et al (1994) ${ }^{12}$ has also conjunctival involvement in $82 \%$ cases with SJS. In the acute stage, catarrhal type of conjunctivitis was the commonest and was manifested in 50\% study population. This is in concordance with the findings of a study conducted by different investigators like Duggan and Gaines (1951), ${ }^{8}$ Howard (1963) ${ }^{17}$ and Storm (1966) ${ }^{4}$ in which at least $10 \%$ showed mucopurulent conjunctivitis.

Misra and Chandi Das (1968) ${ }^{18}$ described cases of SJS with bivalent severe bulbar, tarsal congestion of conjunctivitis with profuse mucopurulent conjunctivitis which responds with terramycin eye ointment and frequent irrigation of eyes.

Membranous conjunctivitis was shown by 9 (17.3\%). Other acute manifestation like conjunctival chemosis was shown by $9.6 \%$. It was noted more in the patients who had taken sulpha drugs.

\section{Types of Conjunctivitis in the Acute Stage}

In this study, commonest type of conjunctivitis is catarrhal conjunctivitis (50\%) (Table 5). This was in correspondence with the findings of a study conducted by Duggan and Gaines (1951), ${ }^{8}$ Howard (1963) ${ }^{17}$ and Storm (1966). ${ }^{4}$

\section{Symblepharon in Acute Stage}

A study at Tan Tock Seng Hospital, Singapore reported 85 patients out of 117 patients who were being SJS and symblepharon were shown by $6(14 \%)$ in acute period. 10 showed mucopurulent conjunctivitis $(19.2 \%)$. In a study by Misra and Chandi Das (1968) ${ }^{18}$ described a case of SJS with bivalent severe bulbar and tarsal congestion of conjunctivitis with profuse mucopurulent conjunctivitis, which responded well to terramycin eye ointment and frequent irrigation of eyes.

\section{Membranous Conjunctivitis}

9 patients showed membranous conjunctivitis (17.3\%), pseudomembranous conjunctivitis has been reported by Wolff (1949). ${ }^{26}$ In this study 5 patients (9.6\%) showed other types of conjunctivitis.

\section{Corneal involvement in Acute Stage}

Corneal involvement erosions were shown by 9 (17.3\%) and Superficial Punctuate Epithelial Erosions (SPE) were shown by $7(13.6 \%)$ of the total study population. Howard (1963) and Wilkins et al (1992) ${ }^{20}$ reported that corneal erosions and ulceration occur in the acute stage of SJS.

Punctuate Epithelial Erosions (PEE) were shown by 12 (23.3\%) and SPEE were shown by 7 (13.6\%). A study conducted at LV Prasad Eye Institute, Hyderabad, revealed SPEE as noticed in 51 patients out of 95 (Misra and Chandi Das, 1968). ${ }^{18}$
Corneal opacity were noted in 3 (5.7\%) patients, uncommonly corneal opacity occur during acute phase. Among these, three cases with initial or very early ophthalmic interference could not succeed. But Ward et al (2010) ${ }^{27}$ in their series mentioned destruction of the corneal limbal stem cells was perhaps the most due. Consequences of the aforementioned pathologies can lead to vascularisation and thickening of the corneal epithelium.

The incidence of acute corneal corrosion presented in 9 patients were reduced to 3 in the follow-up period and 12 of patients presented with PEE were reduced to 5 in the followup period followed by SPEE. This shows active and early ophthalmologic intervention and meticulous treatment regimen can reduce the corneal involvement to certain extent. Chi-square test conducted in this group $(\mathrm{p}<0.001)$ showed significant reduction in the incidence of corneal involvement in the follow-up period.

A hospital-based other studies (Hallgren et al, 2003) ${ }^{28}$ revealed more adverse reactions to Antiepileptics. In our study showed drug toxicity to NSAIDS (Brufen) as $3(5.8 \%)$ and other groups of drugs as $5(9.6 \%)$. A study conducted by Roujeau et al (1995) ${ }^{1}$ on 245 hospitalised SJS patients also showed risk factor as oral intake of NASIDS $80 \%$.

About the acute ophthalmic manifestation, ocular involvement was very high in this study as similar to a hospital-based study conducted at Taiwan between 1988 2004 (Yip, 2007) ${ }^{7}$ on attackers of SJS/ Epidermal necrolysis of 207 patients showed high ophthalmic involvement (81.3\%).

Acute ophthalmic manifestation like acute stage conjunctivitis was the commonest manifestation and was seen in 50 patients $(96.2 \%)$, higher than other study (82\%) (Yip, 2007). ${ }^{7}$

The acute stage catarrhal type of conjunctivitis was the commonest and was seen in $50 \%$ of the total population of the present study. This was in concordance with the earlier study conducted by Duggan and Gaines (1951). ${ }^{8}$ In our study, Mucopurulent Conjunctivitis (MPC) reported in 19.2\%, similar to other studies conducted by Misra and Chandi Das (1968), ${ }^{18}$ which revealed cases of SJS with profuse mucopurulent conjunctivitis which responds with terramycin eye ointment and frequent irrigation of eyes.

Discussing about the laterality of conjunctival involvement, it seems bilateral i.e. frequencies in right and left eyes is $100 \%$ as similar to a study conducted by Ting and Adam (1985). ${ }^{14}$

A hospital-based study conducted by Wilkins et al $(1992)^{20}$ says corneal erosions and ulceration occurs in acute stage of SJS. In this study, punctate epithelial erosions were shown by $12(23.1 \%)$.

Corneal opacity was noted by $3(5.7 \%)$. But in these three cases, initial or very early ophthalmic interference could not succeed. But a study conducted by De Rojas et al (2007)29 reported destruction of the corneal limbal stem cells, perhaps the most due consequences of the aforementioned pathologies and can lead to vascularisation and corneal epithelial thickening.

Thus, the present study emphasises on the importance of meticulous screening on ocular manifestation in the acute phase and if offered proper treatment modalities can minimise the visual impairment due to ophthalmic morbidities caused by Stevens-Johnson Syndrome. 


\section{CONCLUSION}

SJS is a life-threatening illness with high incidence of ocular involvement. Conjunctivitis is the commonest acute ophthalmic manifestation. Early interference and active treatment regimen can reduce the events and further corneal involvement.

\section{REFERENCES}

[1] Roujeau JC, Kelly JP, Naldi L, et al. Medication use and the risk of Stevens-Johnson Syndrome or toxic epidermal necrolysis. N Engl J Med 1995;333(24):1600-7.

[2] Schopf E, Stuhmer A, Rzany B, et al. Toxic epidermal necrolysis and Stevens-Johnson syndrome. An epidemiologic study from West Germany. Arch Dermatol 1991;127(6):839-42.

[3] Ikeda H, Takahashi Y, Yamazaki E, et al. HLA Class I markers in Japanese patients with carbamazepine induced cutaneous adverse reactions. Epilepsia 2010;51(2):297-300.

[4] Strom J. Ocular symptoms in febrile mucocutaneous reactions (ectodermosis erosiva pluriorificialis, Stevens-Johnson's syndrome, mucocutaneous-ocular syndrome etc.). Acta Ophthalmol (Copenh) 1966;44(3):411-4.

[5] Power WJ, Ghoraishi M, Merayo-Lloves J, et al. Analysis of acute ophthalmic manifestations of erythema multiforme/Stevens-Johnson syndrome/ toxic epidermal necrolysis disease spectrum. Ophthalmology 1995;102(11):1669-76.

[6] Chang HJ, Wang N, Qman VP, et al. Fever, mild elevation in liver function tests, leucopenia of carbamazepine induced anticonvulsants hypersensitivity syndrome. Neurol India 2011;59(1):72-3.

[7] Yip LW, Thong BY, Lim J, et al. Ocular manifestation and complications of SJS toxic epidermal necrolysis: an Asian series. Allergy 2007;62(5):527-31.

[8] Duggan JW, Gaines SR. The ocular complications of erythema exudativum multiforme. Am J Ophthalmol 1951;34(2 Pt 1):189-97.

[9] Pushker N, Tandon R, Vajpayee RB. Stevens-Johnson syndrome in India: risk factors, ocular manifestations and management. Ophthalmologica 2000;214(4):2858.

[10] Thomas BA. The so-called Steven Johnson syndrome. Br Med J 1950;1(4667):1393-7.

[11] Pohloja S, Horsmanheimo A. Keratitis sicca After erythema exudativum multiforme caused by PAS (Case report). Acta Ophthalmologia 1966;44(3):415-9.

[12] Stewart MG, Duncan NO 3rd, Franklin DJ, et al. Head and neck manifestation of erythema multiforme in children. Otolaryngol Head Neck Surg 1994;111(3 Pt 1):236-42.

[13] Ashby DW, Lazar T. Erythema multiforme exudativum major (Stevens-Johnson syndrome). Lancet 1951;1 (6664):1091-5.
[14] Ting HC, Adam BA. Steven-Johnson syndrome. A review of 34 cases. Int J Dermatol 1985;24(9):587-91.

[15] Bianchine JR, Macaraeg PV Jr, Lasagna L, et al. Drug as etiologic factors in the Steven-Johnson syndrome. Am J Med 1968;44(3):390-405.

[16] Foster CS, Fong LP, Azar D, et al. Episodic conjunctival inflammation after Stevens-Johnson syndrome. Ophthalmology 1988;95(4):453-62.

[17] Howard GM. The Steven-Johnson syndrome. Ocular prognosis and treatment. Am J Ophthal 1963;55:893900.

[18] Misra K, Chandi Das. Ectodermosis erosive rluri orificialis. J All India Ophthal Soc 1968;16:152-3.

[19] Champion RH, Burton JL, Burns DA, et al. Text book of dermatology. Vol. 4. $6^{\text {th }}$ edn. Black-Well Science 1998: p. 3375-8.

[20] Wilkins J, Morrison L, White CR Jr. Oculo-cutaneous manifestations of the erythema multiforme/StevensJohnson Syndrome/toxic epidermal necrolysis spectrum. Dermatol Clin 1992;10(3):571-82.

[21] Albert DM, Jakobie FA, Azar DT, et al. Principles and practice of ophthalmology. Vol. 2. $2^{\text {nd }}$ edn. WB Saunders Company 1994: p. 821-4.

[22] Shah SS, Doshi HV, Kothari UR. Steven-Johnson syndrome: a study of 17 cases. Indian J Dermatol Venereol Leprol 1977;43(1):6-11.

[23] Sarkari NS, Nigam DK, Verma BMD, et al. StevenJohnson syndrome (an analysis of 20 cases). J Assoc Physicians India 1972;24:917-24.

[24] Shaffer B, Morris P. Severe erythema multiforme of the pluriorificial type (Steven - Johnson syndrome) resulting in blindness in a patient treated with trimethadione (tridione). Pediatrics 1948;2(1):30-4.

[25] Huff JC, Weston WL, Tonnesen MG. Erythema multi forme: a critical review of characteristics, diagnostic criteria and causes. J Am Acad Dermatol 1983;8(6):763-75.

[26] Wolff JE. Ocular complications in erythema exudativum multiforme with mucous membrane lesions. Br J Ophthalmol 1949;33(2):110-20.

[27] Ward KE, Archambault R, Mersfelder TL. Severe adverse skin reactions to non-steriodal antiinflammatory drug: a review of the literature. American Journal of Health System Pharmacy 2010;67(3):206-13.

[28] Hallgren J, TengVall-Linder M, Persson M, et al. SJS associated with ciprofloxacin: a review of adverse cutaneous events reported in Sweden as associated with this drug. J Am Acad Dermatol 2003;49(Suppl 5):S267-S9.

[29] De Rojas MV, Dart JK, Saw VP. The natural history of Steven Johnson syndrome: patterns of chronic ocular disease and the role of systemic immunosuppressive therapy. Br J Ophthalmol 2007;91(8):1048-53. 